CERN-TH.7403/94

SHEP 94/95-04

\title{
The Renormalization Group and Two Dimensional Multicritical Effective Scalar Field Theory
}

\author{
Tim R. Morris \\ CERN TH-Division \\ CH-1211 Geneva 23 \\ Switzerland*
}

\begin{abstract}
Direct verification of the existence of an infinite set of multicritical non-perturbative FPs (Fixed Points) for a single scalar field in two dimensions, is in practice well outside the capabilities of the present standard approximate non-perturbative methods. We apply a derivative expansion of the exact RG (Renormalization Group) equations in a form which allows the corresponding FP equations to appear as non-linear eigenvalue equations for the anomalous scaling dimension $\eta$. At zeroth order, only continuum limits based on critical sine-Gordon models, are accessible. At second order in derivatives, we perform a general search over all $\eta \geq .02$, finding the expected first ten FPs, and only these. For each of these we verify the correct relevant qualitative behaviour, and compute critical exponents, and the dimensions of up to the first ten lowest dimension operators. Depending on the quantity, our lowest order approximate description agrees with CFT (Conformal Field Theory) with an accuracy between $0.2 \%$ and $33 \%$; this requires however that certain irrelevant operators that are total derivatives in the CFT are associated with ones that are not total derivatives in the scalar field theory.
\end{abstract}

CERN-TH.7403/94

SHEP 94/95-04

hep-th/9410141

October, 1994.

* On Leave from Southampton University, U.K. (Address after 1/10/94). 
Circumstantial evidence strongly suggests that there exists an infinite set of multicritical non-perturbative FPs for a single scalar field in two dimensions, corresponding to the universality classes of multicritical Ising models, equivalently to the diagonal invariants of the unitary minimal $(p, p+1)$ conformal models with $p=3,4, \cdots[1][2]$, however direct verification of these facts is in practice well outside the capabilities of the standard approximate non-perturbative methods: lattice Monte Carlo, resummations of weak or strong coupling perturbation theory and the epsilon expansion. (The impracticableness of the epsilon expansion for higher $p$ is covered in ref.[3], implying similar difficulties in weak coupling perturbation theory, while lattice methods suffer from difficulties of locating and accurately computing the multicritical points in the at least $p-2$ dimensional bare coupling constant space). In this letter we demonstrate directly that these multicritical points do exist - by an approximation scheme which is reviewed below. Our main motivation for the present letter is to show that the approximation scheme, which we presented and applied to three and four dimensional scalar field theory in refs. [5][6], is powerful enough, in fact to automatically uncover, and reliably describe, the expected much richer set of non-perturbative theories in two dimensions. Many of our operator dimensions are computed to at least the same accuracy as that of the most relevant operator - very much in contrast to all the above approximation methods, suggesting that our equations may also provide a reliable description well away from FPs. These facts are certainly encouraging for our ambition of developing a reliable and accurate analytic approximation method of general applicability to non-perturbative quantum field theory[4]-[6].

As mentioned in the abstract we use the approximation scheme carried to the lowest sensible order, to verify that each of the first ten multicritical points $(p=3, \cdots, 12)$ have the correct qualitative behaviour - e.g. shape of potential, number of relevant directions, expected parities under $\varphi \leftrightarrow-\varphi$ etc. - and compute critical exponents, and scaling dimensions of up to the first ten lowest dimension (integrated) operators. All of the $\sim 100$ quantities agree with CFT to (sometimes much better than) 33\%, with a weak improvement in accuracy with increasing multicriticality. However, we find that some integrated irrelevant operators appear in the scalar field theory spectrum, which can only be identified with operators which are total derivatives 
in the CFT. It appears to be an interesting challenge to understand why these integrated operators do not vanish.

We start with a review of the method[5]. We wish to formulate quantum field theory in a way in which it is obvious that our approximations are renormalizable[4]. We do so by never needing to determine bare quantities, such as a bare action $S_{\Lambda_{0}}[\varphi]$, or equivalently counterterms. In this letter we discuss only quantum field theories with no low energy mass scale. (The generalisation to massive ones is in principle straightforward). If indeed such theories are independent of the cutoff $\Lambda_{0}$ then the (renormalized) theories have scale invariance. As is well known this is achieved only by assigning scaling dimensions to the operators, which are different from their naïve dimensions in general. Thus for a single component scalar field $\varphi(x)$ in $D$ (euclidean) dimensions we have that its scaling dimension is $d_{\varphi}=\frac{1}{2}(D-2+\eta)$, where $\eta$ is the anomalous scaling dimension. For example, this implies by dimensional analysis that propagators, as a function of momentum $q$, appear as

$$
<\varphi \varphi>\propto 1 / q^{2-\eta}
$$

To write the condition that the theories be scale invariant, and to formulate an efficacious approximation scheme, it is helpful to introduce a (low energy) scale ${ }^{1} \Lambda$. Now by dimensions, (1) appears as

$$
<\varphi \varphi>\propto \frac{1}{\Lambda^{2-\eta}} f\left(q^{2} / \Lambda^{2}\right)
$$

for some function $f$. We will require that $\Lambda$ is introduced in such a way that $f$ can be Taylor expanded for small $q^{2} / \Lambda^{2}$, because our approximation will follow from a momentum expansion (equivalently derivative expansion) by dropping terms beyond some maximum order. This requirement implies that the infrared singularity in (1) has been smoothly regulated. Therefore $\Lambda$ is equivalent to a smooth infrared cutoff. We introduce it into the partition function $Z[J]$ as $C(q, \Lambda)$, satisfying $C(q, \Lambda) \rightarrow 0$ as $q \rightarrow 0$, by writing $S_{\Lambda_{0}}[\varphi] \mapsto S_{\Lambda_{0}}[\varphi]+\frac{1}{2} \varphi \cdot C^{-1} \cdot \varphi$, where only $C$ depends on $\Lambda$. From this it is straightforward to write down a differential equation for $Z[J]$ with respect to $\Lambda$. It turns out however to be helpful to transform this to a differential

1 In the Wilson sense this is the intermediate cutoff at which one defines the effective action[7]. This connection is developed in ref.[4]. 
equation for the Legendre effective action. (One important reason is because the integrals involved will converge, with our choice of $C$, only if the full self-energy is used - expanded as a power series to the prescribed maximum order). In terms of this, after expressing $\varphi, C$ and $q$ as dimensionless quantities ${ }^{2}$ using $\Lambda$, we find[5]

$$
\begin{aligned}
& \left(\frac{\partial}{\partial t}+d_{\varphi} \Delta_{\varphi}+\Delta_{\partial}-D\right) \Gamma[\varphi]= \\
& \quad-\zeta \int_{0}^{\infty} d q q^{D-1}\left(\frac{q}{C\left(q^{2}\right)} \frac{\partial C\left(q^{2}\right)}{\partial q}+2-\eta\right)\left\langle\left[1+C \cdot \frac{\delta^{2} \Gamma}{\delta \varphi \delta \varphi}\right]^{-1}(\mathbf{q},-\mathbf{q})\right\rangle
\end{aligned}
$$

Here $t=\ln (\mu / \Lambda)$ with $\mu$ some arbitrary reference scale. The angle brackets refer to an average over all directions of the vector q. $\zeta$ is a normalization factor, introduced for convenience, by a numerical rescaling. $\Delta_{\varphi}=\varphi \cdot \frac{\delta}{\delta \varphi}$ counts the number of fields in a given vertex, and $\Delta_{\partial}$ counts the number of derivatives in a given vertex[5].

The requirement of scale invariance is now simply given by $\frac{\partial}{\partial t} \Gamma[\varphi]=0$. Substituting this and a derivative expansion

$\Gamma[\varphi]=\int d^{D} x\left\{V(\varphi, t)+\frac{1}{2}\left(\partial_{\mu} \varphi\right)^{2} K(\varphi, t)+\left(\partial_{\mu} \varphi\right)^{4} H_{1}(\varphi, t)+(\square \varphi)^{2} H_{2}(\varphi, t)+\cdots\right\}$

into (3) and expanding the RHS (Right Hand Side) to some maximum order in derivatives yields $n$ coupled second order non-linear ordinary differential equations for the fixed point coefficient functions $V(\varphi), K(\varphi), H_{1}(\varphi), H_{2}(\varphi), \ldots$, where $n$ is the number of undetermined coefficient functions. As such, we expect at first sight there to be a $2 n$ parameter set of solutions. In fact, generally there are only a discrete set of possible solutions. Of course only a discrete set of solutions is generally expected on physical grounds: they correspond to the possible massless continuum limits (continuous phase transitions) with the prescribed field content. Mathematically this arises because nearly all choices of BCs (Boundary Conditions) for the differential equations lead to solutions with singular behaviour at some finite real value of the field $\varphi[5][6]$. Another way of seeing why only a discrete set of solutions is allowed is as follows. We take for simplicity a theory with $Z_{2}$ symmetry $\varphi \leftrightarrow-\varphi$, and assume $d_{\varphi} \neq 0$. (See later for what happens when these conditions are relaxed). In this case there are $n$ BCs given by symmetry - namely that the first

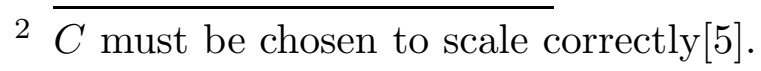


derivative of the coefficient functions $\left(V^{\prime}(\varphi), K^{\prime}(\varphi)\right.$, etc.) vanish at $\varphi=0$, while a further $n$ BCs are given by the behaviour of the coefficient functions for large $\varphi$ which are determined, up to a proportionality constant, by dimensional analysis assuming that in this limit $\Lambda$ can be neglected:

$$
V(\varphi) \propto \varphi^{D / d_{\varphi}}, \quad K(\varphi) \propto \varphi^{(D-2) / d_{\varphi}-2}, \quad \cdots \quad \text { as } \quad \varphi \rightarrow \infty
$$

Since these $2 n$ conditions $^{3}$ are imposed on a $2 n$ parameter set, we again expect only a discrete set of solutions. Actually this is not the whole story because the parameter $\eta$ in $(3)$ (with $\frac{\partial}{\partial t} \Gamma[\varphi]=0$ ) must still be determined. Generally this is only possible if there is a reparametrization invariance of the RG equations. This turns the equations into non-linear eigenvalue equations for $\eta$. (To see this, use the invariance to fix an extra condition). Clearly it is important that such an invariance is preserved by the approximation scheme. We can do this if we choose $C\left(q^{2}\right)$ to be homogeneous in $q$, i.e. $C\left(q^{2}\right) \propto q^{2 \kappa}$, since then a reparametrization invariance of the equations exists according to the following (non-physical) scaling dimensions:

$$
\begin{gathered}
{\left[\partial_{\mu}\right]=\left[q_{\mu}\right]=1, \quad[\varphi]=\kappa+D / 2,} \\
\text { hence } \quad[V]=D, \quad[K]=-2(\kappa+1), \quad\left[H_{i}\right]=-D-4(\kappa+1),
\end{gathered}
$$

and this is clearly not affected by neglecting higher derivative terms. The value of $\kappa$ may be determined uniquely, by considerations of convergence, to be the smallest integer larger than $D / 2-1[5]$.

The operator spectrum may be determined by linearization of (3) about the FP solutions. By separation of variables the perturbations are of the form $\delta V(\varphi, t)=$ $\varepsilon(\mu / \Lambda)^{\lambda} v(\varphi), \delta K(\varphi, t)=\varepsilon(\mu / \Lambda)^{\lambda} k(\varphi), \delta H_{i}(\varphi, t)=\varepsilon(\mu / \Lambda)^{\lambda} h_{i}(\varphi)$, etc, for some functions $v, k, h_{i}$ etc, where $\varepsilon$ is infinitessimal and $\lambda$ appears as an eigenvalue for the linearized equations. In fact $\lambda$ is quantized since we have again $2 n+1$ constraints: $n$ from symmetry $\left(v^{\prime}(0)=0, k^{\prime}(0)=0, \cdots\right.$ for even eigenfunctions, or $v(0)=0, k(0)=0, \cdots$ for odd eigenfunctions), $n$ from dimensional analysis $\left(v(\varphi) \propto \varphi^{(D-\lambda) / d_{\varphi}}, k(\varphi) \propto \varphi^{(D-2-\lambda) / d_{\varphi}-2}, \cdots\right.$ as $\varphi \rightarrow \infty$, again providing $\Lambda$ can be ignored in this limit), and one from a normalization constraint allowed by linearity. $\varepsilon \mu^{\lambda}$ plays the rôle of an infinitessimal coupling constant of dimension $\lambda$, conjugate

$3 \overline{\text { If } Z_{2} \text { symmetry is dropped, }}$ (5) for $\varphi \rightarrow \pm \infty$ provides all $2 n$ conditions. 
to an integrated operator of the form $\gamma[\varphi]=\int d^{D} x\left\{v(\varphi)+\frac{1}{2} k(\varphi)\left(\partial_{\mu} \varphi\right)^{2}+\cdots\right\}$. Note that this implies that the operator itself has dimension $D-\lambda$.

In interpreting the operator spectrum it is important to discard any redundant operators[8]. These are operators of the form[5]

$$
\gamma[\varphi]=\int d^{D} x F_{\mathbf{x}}[\varphi] \delta \Gamma[\varphi] / \delta \varphi(\mathbf{x})
$$

corresponding to reparametrizations of the effective action. They have no physical significance, and no well-defined scaling dimension since it depends on the details of the RG used[8]. Since we already have $2 n+1$ constraints, the requirement (7) will overconstrain the problem leading to no solutions unless the redundant operators exist for special reasons (viz. symmetries). We know of two such operators. One is the operator corresponding to the symmetry (6), which thus has (with some arbitrary normalisation) $F_{\mathbf{x}}[\varphi]=-x^{\mu} \partial_{\mu} \varphi(\mathbf{x})-(\kappa+D / 2) \varphi(\mathbf{x}), v(\varphi)=D V(\varphi)-$ $(\kappa+D / 2) \varphi V^{\prime}(\varphi), k(\varphi)=-2(\kappa+1) K(\varphi)-(\kappa+D / 2) \varphi K^{\prime}(\varphi), \cdots$, even parity, and eigenvalue $\lambda=0$. And the other[8] corresponds to the $\varphi$ translation symmetry of the unscaled RG equations. It has $F_{\mathbf{x}}[\varphi]=1, v(\varphi)=V^{\prime}(\varphi), k(\varphi)=K^{\prime}(\varphi), \cdots$, and odd parity. By operating with $\int d^{D} x \delta / \delta \varphi(\mathbf{x})$ on $(3)$, it can be seen that, to any order of the derivative expansion, $\lambda=d_{\varphi}$, as expected on dimensional grounds.

From now on we set $D=2$. This implies $\kappa=1$. To $O\left(\partial^{2}\right)$ we drop all derivatives higher than second on the RHS of (3). Since the higher FP coefficient functions $H_{i}, \cdots$, then satisfy linear equations given by the LHS of (3) with $\frac{\partial}{\partial t} \Gamma[\varphi]=0$, they must vanish, otherwise they have singularities at $\varphi=0$ (for general values of $\eta$ ) which is unacceptable. Thus the inverse operator in (3) is the same as that computed in ref.[5]. Performing the average, the $q$ integral, choosing $\zeta=1 / 4$, and matching both sides of $(3)$ we find for $V(\varphi, t)$ and $K(\varphi, t)$ :

$$
\begin{gathered}
\frac{\partial V}{\partial t}+\frac{\eta}{2} \varphi V^{\prime}-2 V=-\left(1-\frac{\eta}{4}\right) P_{K}\left(V^{\prime \prime}\right) \\
\text { and } \frac{\partial K}{\partial t}+\frac{\eta}{2} \varphi K^{\prime}+\eta K=\left(1-\frac{\eta}{4}\right)\left\{P _ { K } ( V ^ { \prime \prime } ) \left[\frac{V^{\prime \prime} K^{\prime \prime}-4 V^{\prime \prime \prime} K^{\prime}}{2\left(V^{\prime \prime 2}-4 K\right)}\right.\right. \\
\left.+\frac{2 K V^{\prime \prime} V^{\prime \prime \prime}{ }^{2}+13 V^{\prime \prime} K^{\prime 2}-40 K K^{\prime} V^{\prime \prime \prime}}{2\left(V^{\prime \prime}{ }^{2}-4 K\right)^{2}}+10 K \frac{K V^{\prime \prime} V^{\prime \prime \prime}-4 K K^{\prime} V^{\prime \prime \prime}+V^{\prime \prime} K^{\prime 2}}{\left(V^{\prime \prime}{ }^{2}-4 K\right)^{3}}\right] \\
\left.-\frac{12 K K^{\prime \prime}+11 K^{\prime 2}}{24 K\left(V^{\prime \prime 2}-4 K\right)}+\frac{20 K^{\prime} V^{\prime \prime} V^{\prime \prime \prime}-11 K V^{\prime \prime \prime}{ }^{2}-44 K^{\prime 2}}{6\left(V^{\prime \prime 2}-4 K\right)^{2}}-10 K \frac{K V^{\prime \prime \prime}{ }^{2}-K^{\prime} V^{\prime \prime} V^{\prime \prime \prime}+K^{\prime 2}}{\left(V^{\prime \prime 2}-4 K\right)^{3}}\right\}
\end{gathered}
$$




$$
\text { where } \quad \begin{aligned}
P_{b}(a) & =\frac{\tanh ^{-1} \sqrt{1-4 b / a^{2}}}{\sqrt{a^{2}-4 b}} \quad \text { if } & a^{2}>4 b \\
& =\frac{\tan ^{-1} \sqrt{4 b / a^{2}-1}}{\sqrt{4 b-a^{2}}} \quad \text { if } & a^{2}<4 b,
\end{aligned}
$$

and $\tan ^{-1}$ is taken in the range $0 \leq \tan ^{-1} \leq \pi$. Eqns. (8) hold true only if

$$
K>0 \quad \text { and } \quad V^{\prime \prime}>-2 \sqrt{K},
$$

for otherwise the integrals diverge at unphysical poles. These conditions are sufficient to ensure that the obvious physical stability requirements are satisfied.

Consider now the $O\left(\partial^{0}\right)$ case, where the RHS of $(8 b)$ is also dropped. Then $\eta=0$, since otherwise the FP $K$ solution is singular: $K(\varphi) \propto 1 / \varphi^{2}$. With $\eta=0, K(\varphi)$ is not determined. We will here simply assume that $K(\varphi) \equiv 1$ is a good approximation. The remaining FP equation is now $2 V(\varphi)=P_{1}\left(V^{\prime \prime}\right)$. Since $P_{1}(a)$ is a positive monotonically decreasing function which diverges at $a=-2$, the 'potential' $U(x)=$ $-\int^{x} d y P_{1}^{-1}(2 y)$ is bounded below with a single stationary point. If we think of $V$ as position $x$, and $\varphi$ as the time $\tau$, then we see that, apart from the Gaussian FP $(V(\varphi) \equiv \pi / 8), V(\varphi)$ has only periodic solutions, as follows from Newton's equation $d^{2} x / d \tau^{2}=-\partial U / \partial x$. These correspond to the semi-infinite line of critical sineGordon models (that is circular bosons with a radius tunable to any value larger than some minimum radius). It would take us too far from our present purpose however to flesh this out. Since $d_{\varphi}=0$, eqn. (5) does not apply; in fact the unscaled $V(\varphi)$ is nowhere independent of $\Lambda$ and vanishes in the limit $\Lambda \rightarrow 0$. (Incidentally, the $d_{\varphi} \varphi V^{\prime}(\varphi)$ term in $(8 a)$ corresponds, in the above Newtonian analogy, to a negative friction term proportional to velocity and to the time. Physically it is then easy to understand that this term is responsible for generic singular behaviour and that the careful balancing act (5) is necessary if this is to be avoided.)

Returning to the full $O\left(\partial^{2}\right)$ equations, we substitute a differentiated $(8 a)$ into the $V^{\prime \prime \prime}$ terms in (8b) to turn (8) manifestly into a pair of second order differential equations. We analyse their FPs by the numerical method of relaxation[9]. We can find the approximate $p=3$ (Ising model) solution and its eigenvalue $\eta=\eta_{I}$ by using a full set of BCs and supplying an initial guess (e.g. the $D=3$ Ising solution[5]), 


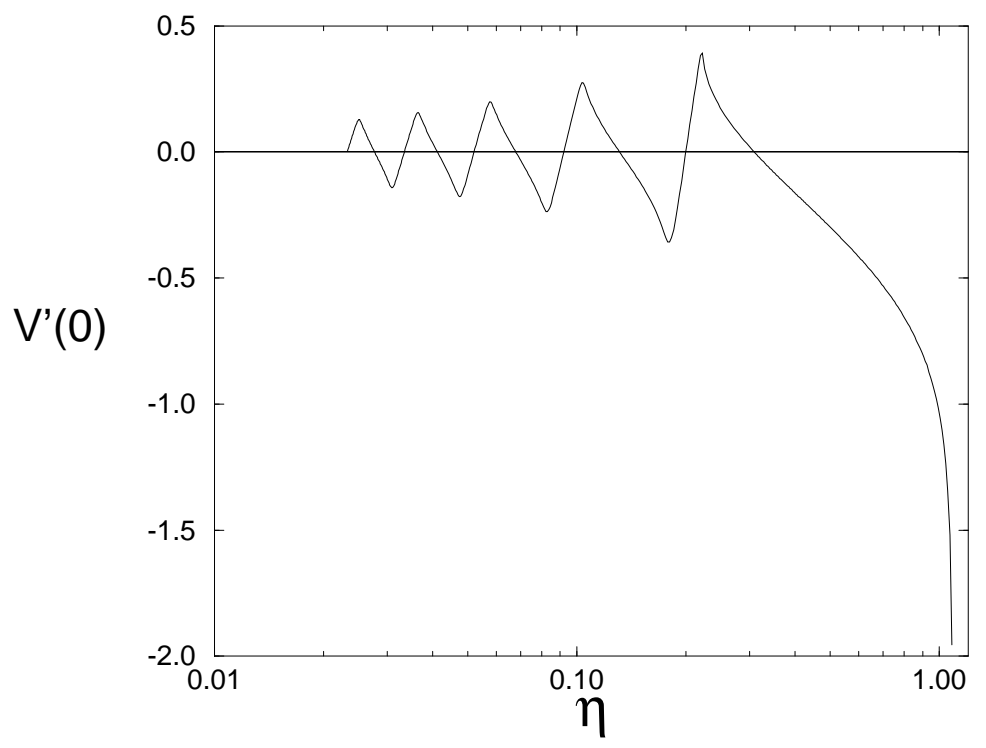

Fig.1. $V^{\prime}(0) \stackrel{\varepsilon}{\text { versus }} \eta$. Note that no solution was found for $\eta>1.08$. The $p=3 \cdots 12$ FPs correspond to the 10 places where $V^{\prime}(0)$ crosses the axis.

however finding the other solutions this way is much harder. (More details of the numerics are given in the appendix). Anyway, we want to demonstrate that for $d_{\varphi} \neq 0$, the eqns. (8) have no other FPs except the $(p, p+1)$ points. This is the expected result, by Zamolodchikov's $c$-theorem[10], if the FP can be the result of flow from a Landau Ginsburg Lagrangian based about the $c=1$ ultraviolet Gaussian FP. In fact we will here strictly only show that no other connected FPs exist in the following sense: Starting from our approximate Ising FP, we relax the BCs by dropping the requirement $V^{\prime}(0)=0$ and instead determine a unique solution as a function of $\eta$ by using relaxation while gradually changing $\eta$ away from $\eta_{I}$. The result is plotted in fig. 1 for all $\eta \geq .02$. Each time $V^{\prime}(0)$ crosses the axis we store the solution and use this as a guess for 'polishing' by relaxation with the full set of BCs. (A more conclusive demonstration that there are no other FPs would involve relaxing e.g. the $K^{\prime}(0)$ condition also, and searching a two dimensional parameter space). Similarly, using relaxed BCs and starting from the exactly known operators given earlier or below, we can determine all $^{4}$ the even and odd parity eigenoperators and eigenvalues at any of the FPs. Here one must be careful that the normalisation

4 Here we can be confident that all solutions are connected since we do not expect or find at any point a two dimensional linear space of solutions to the relaxed BCs. 
condition can be satisfied at any value $\lambda$. We chose $v^{\prime}(0)^{2}+v(0)^{2}=1$, dropping the requirement $v^{\prime}(0)=0(v(0)=0)$ for the even(odd) parity solutions.

Some results are displayed in the table below. The dimensions and parities of the two most relevant operators are not displayed since the unit operator $v(\varphi)=1$, $k=h_{i}=\cdots=0$, has dimension 0 and even parity for any FP and to any order in the derivative expansion, while the field itself $v(\varphi)=\varphi, k=h_{i}=\cdots=0$, always has dimension $d_{\varphi}(=\eta / 2$ in this case) and odd parity. (These follow easily from the vanishing of the RHS of (3) linearized about the FP). The correlation length critical exponent is given by $\nu=1 / \lambda$ where $\lambda$ is the largest even parity eigenvalue, excluding the unit operator[7]. All numbers were determined to an accuracy greater than that shown. ${ }^{5}$ Also shown in the table are the corresponding results expected from CFT[1][2][11]. These follow from the dimensions $\Delta_{n, m}=\frac{[(p+1) n-p m]^{2}-1}{2 p(p+1)}$ and $Z_{2}$ parities $P_{n, m}=(-)^{(n+1)(p+1)+(m+1) p}$ of the scalar primary fields $\Phi_{n, m}(x), \Phi_{1,1} \equiv 1$ and $\Phi_{2,2} \equiv \varphi$, where $m, n$ are integers in the range $1 \leq n \leq p-1,1 \leq m \leq p$. In addition there are an infinite number of Virasoro descendents, however all but $L_{-1} \tilde{L}_{-1} \Phi_{n, m} \equiv \square \Phi_{n, m}$ have too high a dimension to correspond to the $O\left(\partial^{2}\right)$ results in the table. Thus $2 p-3$ relevant operators are expected $\left(\Phi_{k, k}, k=1, \cdots, p-1\right.$ and $\left.\Phi_{k+1, k}, k=1, \cdots, p-2\right)$, of which $p-1$ are even and $p-2$ are odd. We checked that this is true for all our solutions. In fact, as one can see from the table, these CFT values and our results match unambiguously. As further evidence for the correspondence we note that if the bare Lagrangian is taken to be of Landau Ginsburg type with a potential which is an even polynomial in $\varphi$ of degree $2 p-2$ [1][2], then we might expect the FP Wilson potential to have $p-1$ minima; It does. Examples are shown in fig.2 and ref.[12]. Similarly, the $v(\varphi)$ component of the operators turn out to have the maximum number of nodes expected (c.f. fig.4).

We do not find all the irrelevant operators expected from CFT, which is why there are blank entries in the table. For each $p$ we obtain a cluster of solutions and then a gap in dimensions, much larger than expected from CFT. We report the $p=3,4,5$ cases in detail. For $p=3$, we find the first operator, other than those in the table, is an even operator with $\Delta=20 \pm 2$. (The estimated error is due to truncation in the

$5 \overline{\text { Thus the small dimensions }}$ at the bottom of the table correspond to $\lambda$ 's computed to better than 5 significant figures, and agreeing with CFT to 3 significant figures. 


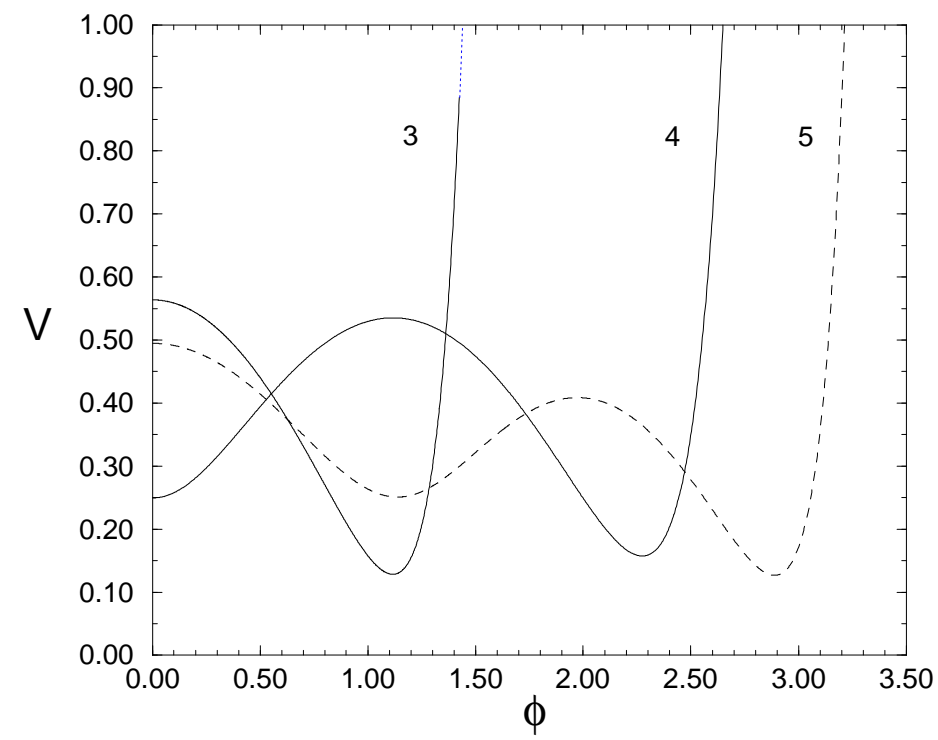

Fig.2. Potentials $V(\varphi)$ for the Ising $(p=3)$, tricritical Ising $(p=4)$, and quadricritical Ising $(p=5)$ fixed points.

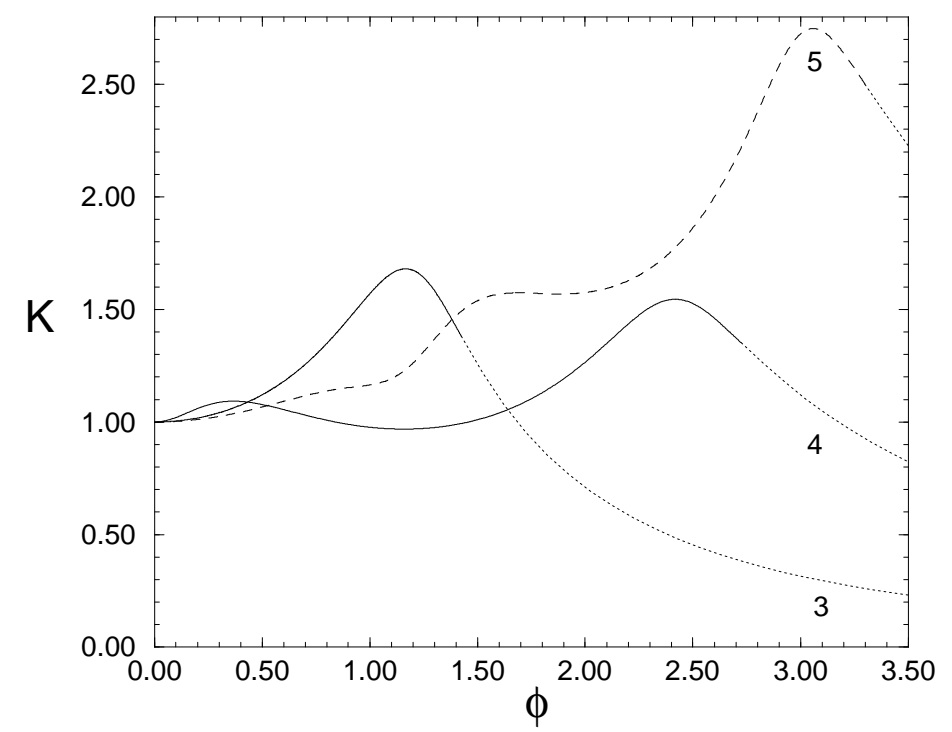

Fig.3. Fixed point kinetic factors $K(\varphi)$ for $p=3,4,5$. As in fig.2, the full solution is shown as a full (or for $p=5$ dashed) line, while the dotted parts of the curve are given by the asymptotic expansion (using $A_{V}$ and $A_{K}$ ). Eqn.(6) has been used to scale the solutions to conventional normalisation $K(0)=1$, from the $K_{\text {asy }}^{\prime \prime}(1)=0$ normalisation. (See appendix). 


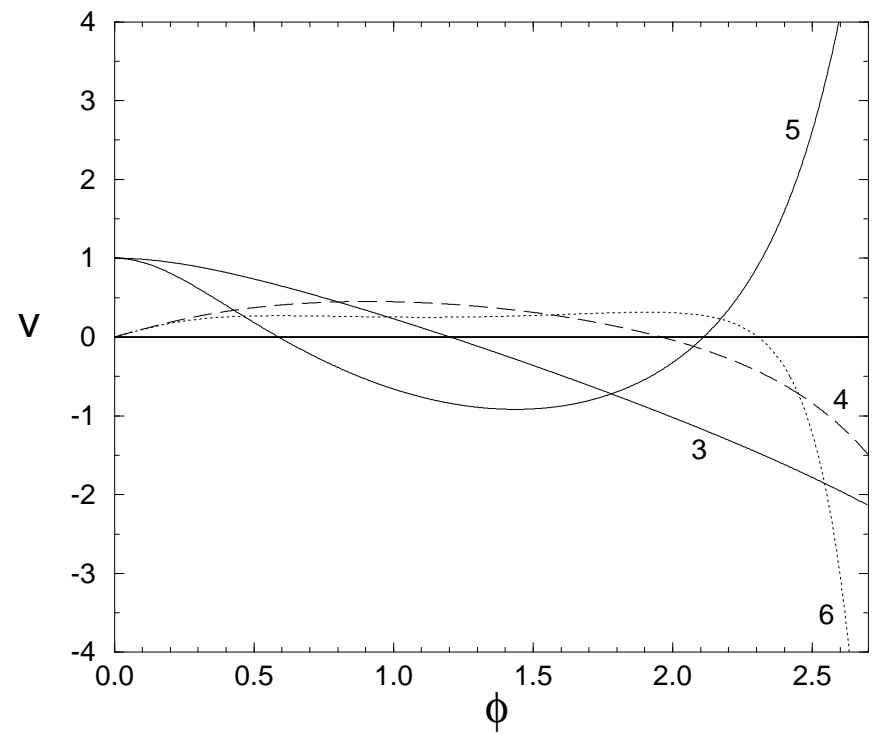

Fig.4. The $v(\varphi)$ component of the $3^{\text {rd }}-6^{\text {th }}$ eigenoperators at the tricritical Ising model fixed point $(p=4)$. As in figs.1 and 2 , the solutions have been scaled to $K(0)=1$ normalisation. Furthermore the even(odd) operators have been normalised so that $v(0)=1\left(v^{\prime}(0)=1\right)$. Note that the $1^{\text {st }}$ operator thus has $v(\varphi) \equiv 1$, and the $2^{\text {nd }}$ has $v(\varphi) \equiv \varphi$. The $3^{\text {rd }}-5^{\text {th }}$ are identified at the bare level with the powers $\varphi^{2}-\varphi^{4}$ in refs.[1][2] (plus lower dimension counterterms of the same $Z_{2}$ symmetry), while the $6^{\text {th }}$ is one of the "puzzling operators".

asymptotic BCs). In the $p=4$ spectrum we find only those operators in the table, in particular no sign of $L_{-2} \tilde{L}_{-2} \Phi_{2,2}$ (dimension and parity $\Delta^{P}=4 \frac{3}{40}^{-}$). In $p=5$, we find an $11^{\text {th }}$ and a $12^{\text {th }}$ operator with $\Delta^{P}=2.61^{+}$and $2.80^{-}$respectively, and then nothing else out to at least $\Delta=8$, and in particular the primary $\Phi_{4,1}\left(\Delta^{P}=6^{+}\right)$ is missing. However, high dimension operators can correspond to operators with many derivatives, and therefore may not appear at our level of approximation.

More puzzling are the integrated operators that are there, but (apparently) should not be because they only match total derivative operators in CFT (given as boldface entries in the table). Thus at $p=3$, the $4^{\text {th }}$ operator's $\Delta^{P}$ matches well with $\square \Phi_{2,2}$, and not with any other operator - in particular the first odd operator that is not a total derivative has $\Delta=6 \frac{1}{8}$. Similarly all the other irrelevant dimensions mentioned, match $\square$ on the relevant CFT operators, with the $11^{\text {th }}\left(12^{\text {th }}\right)$ operators in $p=5$ matching the values $2 \frac{4}{5}^{+}\left(3 \frac{1}{20}^{-}\right)$. Only for the $7^{\text {th }}$ operator in $p=4$, and the $11^{\text {th }}$ and $12^{\text {th }}$ in $p=5$, would alternative assignments be possible within the accuracy 
suggested by the table: to $\Phi_{3,1}\left(3^{+}\right), \Phi_{3,1}\left(2 \frac{4}{5}^{+}\right)$, and $\Phi_{4,2}\left(3 \frac{1}{4}^{+}\right)$respectively.

\begin{tabular}{|c|c|c|c|c|c|c|c|c|c|c|}
\hline$p$ & $\eta$ & $\nu$ & $3^{\text {rd }}$ & $4^{\text {th }}$ & $5^{\text {th }}$ & $6^{\text {th }}$ & $7^{\text {th }}$ & $8^{\text {th }}$ & $9^{\text {th }}$ & $10^{\text {th }}$ \\
\hline 3 & $\begin{array}{l}.309 \\
.25\end{array}$ & $\begin{array}{l}.863 \\
1\end{array}$ & $\begin{array}{l}.841^{+} \\
1^{+}\end{array}$ & $\begin{array}{l}2.61^{-} \\
\mathbf{2 . 1 3}^{-}\end{array}$ & & & & & & \\
\hline 4 & $\begin{array}{l}.200 \\
.15\end{array}$ & $\begin{array}{l}.566 \\
.556\end{array}$ & $\begin{array}{l}.234^{+} \\
.2^{+}\end{array}$ & $\begin{array}{l}.732^{-} \\
.875^{-}\end{array}$ & $\begin{array}{l}1.09^{+} \\
1.2^{+}\end{array}$ & $\begin{array}{l}2.11^{-} \\
\mathbf{2 . 0 8 ^ { - }}\end{array}$ & $\begin{array}{l}2.44^{+} \\
\mathbf{2 . 2}^{+}\end{array}$ & $\begin{array}{l}2.71^{-} \\
\mathbf{2 . 8 8 ^ { - }}\end{array}$ & & \\
\hline 5 & $\begin{array}{l}.131 \\
.1\end{array}$ & $\begin{array}{l}.545 \\
.536\end{array}$ & $\begin{array}{l}.166^{+} \\
.133^{+}\end{array}$ & $\begin{array}{l}.287^{-} \\
.25^{-}\end{array}$ & $\begin{array}{l}.681^{+} \\
.8^{+}\end{array}$ & $\begin{array}{l}.953^{-} \\
1.05^{-}\end{array}$ & $\begin{array}{l}1.26^{+} \\
1.33^{+}\end{array}$ & $\begin{array}{l}2.11^{-} \\
\mathbf{2 . 0 5 ^ { - }}\end{array}$ & $\begin{array}{l}2.16^{+} \\
\mathbf{2 . 1 3}^{+}\end{array}$ & $\begin{array}{l}2.38^{-} \\
\mathbf{2 . 2 5}\end{array}$ \\
\hline 6 & $\begin{array}{l}.0920 \\
.0714\end{array}$ & $\begin{array}{l}.531 \\
.525\end{array}$ & $\begin{array}{l}.117^{+} \\
.0952^{+}\end{array}$ & $\begin{array}{l}.213^{-} \\
.179^{-}\end{array}$ & $\begin{array}{l}.323^{+} \\
.286^{+}\end{array}$ & $\begin{array}{l}.650^{-} \\
.75^{-}\end{array}$ & $\begin{array}{l}.865^{+} \\
.952^{+}\end{array}$ & $\begin{array}{l}1.11^{-} \\
1.18^{-}\end{array}$ & $\begin{array}{l}1.37^{+} \\
1.43^{+}\end{array}$ & $\begin{array}{l}2.08^{-} \\
\mathbf{2 . 0 4}^{-}\end{array}$ \\
\hline 7 & $\begin{array}{l}.0679 \\
.0536\end{array}$ & $\begin{array}{l}.523 \\
.519\end{array}$ & $\begin{array}{l}.0868^{+} \\
.0714^{+}\end{array}$ & $\begin{array}{l}.159^{-} \\
.134^{-}\end{array}$ & $\begin{array}{l}.249^{+} \\
.214^{+}\end{array}$ & $\begin{array}{l}.348^{-} \\
.313^{-}\end{array}$ & $\begin{array}{l}.629^{+} \\
.714^{+}\end{array}$ & $\begin{array}{l}.806^{-} \\
.884^{-}\end{array}$ & $\begin{array}{l}1.01^{+} \\
1.07^{+}\end{array}$ & $\begin{array}{l}1.22^{-} \\
1.28^{-}\end{array}$ \\
\hline 8 & $\begin{array}{l}.0521 \\
.0417\end{array}$ & $\begin{array}{l}.517 \\
.514\end{array}$ & $\begin{array}{l}.0667^{+} \\
.0556^{+}\end{array}$ & $\begin{array}{l}.123^{-} \\
.104^{-}\end{array}$ & $\begin{array}{l}.193^{+} \\
.167^{+}\end{array}$ & $\begin{array}{l}.277^{-} \\
.243^{-}\end{array}$ & $\begin{array}{l}.368^{+} \\
.333^{+}\end{array}$ & $\begin{array}{l}.613^{-} \\
.688^{-}\end{array}$ & $\begin{array}{l}.764^{+} \\
.833^{+}\end{array}$ & $\begin{array}{l}.933^{-} \\
.993^{-}\end{array}$ \\
\hline 9 & $\begin{array}{l}.0412 \\
.0333\end{array}$ & $\begin{array}{l}.514 \\
.511\end{array}$ & $\begin{array}{l}.0529^{+} \\
.0444^{+}\end{array}$ & $\begin{array}{l}.0972^{-} \\
.0833^{-}\end{array}$ & $\begin{array}{l}.154^{+} \\
.133^{+}\end{array}$ & $\begin{array}{l}.221^{-} \\
.194^{-}\end{array}$ & $\begin{array}{l}.299^{+} \\
.267^{+}\end{array}$ & $\begin{array}{l}.383^{-} \\
.350^{-}\end{array}$ & $\begin{array}{l}.601^{+} \\
.667^{+}\end{array}$ & $\begin{array}{l}.733^{-} \\
.794^{-}\end{array}$ \\
\hline 10 & $\begin{array}{l}.0334 \\
.0273\end{array}$ & $\begin{array}{l}.511 \\
.509\end{array}$ & $\begin{array}{l}.0429^{+} \\
.0364^{+}\end{array}$ & $\begin{array}{l}.0790^{-} \\
.0682^{-}\end{array}$ & $\begin{array}{l}.125^{+} \\
.109^{+}\end{array}$ & $\begin{array}{l}.180^{-} \\
.159^{-}\end{array}$ & $\begin{array}{l}.245^{+} \\
.218^{+}\end{array}$ & $\begin{array}{l}.317^{-} \\
.286^{-}\end{array}$ & $\begin{array}{l}.395^{+} \\
.364^{+}\end{array}$ & $\begin{array}{l}.592^{-} \\
.650^{-}\end{array}$ \\
\hline 11 & $\begin{array}{l}.0277 \\
.0227\end{array}$ & $\begin{array}{l}.509 \\
.508\end{array}$ & $\begin{array}{l}.0355^{+} \\
.0303^{+}\end{array}$ & $\begin{array}{l}.0654^{-} \\
.0568^{-}\end{array}$ & $\begin{array}{l}.103^{+} \\
.0909^{+}\end{array}$ & $\begin{array}{l}.150^{-} \\
.133^{-}\end{array}$ & $\begin{array}{l}.203^{+} \\
.182^{+}\end{array}$ & $\begin{array}{l}.265^{-} \\
.239^{-}\end{array}$ & $\begin{array}{l}.332^{+} \\
.303^{+}\end{array}$ & $\begin{array}{l}.405^{-} \\
.375^{-}\end{array}$ \\
\hline 12 & $\begin{array}{l}.0233 \\
.0192\end{array}$ & $\begin{array}{l}.508 \\
.506\end{array}$ & $\begin{array}{l}.0299^{+} \\
.0256^{+}\end{array}$ & $\begin{array}{l}.0550^{-} \\
.0481^{-}\end{array}$ & $\begin{array}{l}.0870^{+} \\
.0769^{+}\end{array}$ & $\begin{array}{l}.126^{-} \\
.112^{-}\end{array}$ & $\begin{array}{l}.172^{+} \\
.154^{+}\end{array}$ & $\begin{array}{l}.224^{-} \\
.202^{-}\end{array}$ & $\begin{array}{l}.282^{+} \\
.256^{+}\end{array}$ & $\begin{array}{l}.345^{-} \\
.317^{-}\end{array}$ \\
\hline
\end{tabular}

Table 1. $\eta, \nu$, and the $\Delta^{P}$ 's of the 10 lowest dimension operators (ordered by increasing dimension), for the first 10 multicritical points. The $O\left(\partial^{2}\right)$ answer is shown in the first row and the associated exact CFT result in the second row. Worst determined number: $\eta$ for $p=4$ (33\% off). Best determined number: $\nu$ for $p=12(0.2 \%$ off $)$. $\eta$ 's accuracy gradually improves from $p=4$ to $p=12$. $\nu$ is worst determined at $p=3(13 \%)$ after which all are determined to error less than $2 \%$ and decreasing with increasing $p$. The worst determined operator dimension is the $3^{\text {rd }}$ at $p=5(25 \%)$ after which errors decrease with increasing $p$ and/or increasing dimension. The dimensions of the first half of the relevant operators are always overestimated while those of the second half are always underestimated.

These puzzling solutions could be discarded if they were redundant, but, as argued below (7), this requirement overconstrains. Indeed, assuming that the most general (Poincaré invariant) redundant operator (7) is of the form $F_{\mathbf{x}}[\varphi]=\alpha x^{\mu} \partial_{\mu} \varphi(\mathbf{x})+$ $f(\varphi(\mathbf{x}))+\cdots$, where $\alpha$ is a constant, $f$ some differentiable function, and the rest results in terms only of $O\left(\partial^{2}\right)$ or greater in $\gamma[\varphi]$, then it is easy to show that (for any dimension $D) \gamma[\varphi]$ is a redundant operator only if $v\left(\varphi_{1}\right) V\left(\varphi_{2}\right)=V\left(\varphi_{1}\right) v\left(\varphi_{2}\right)$ for all 
pairs of stationary points $\varphi_{1}, \varphi_{2}$ of $V$. These relations are not even approximately satisfied by our puzzling operators. Finally, using the cases $p=3,4,5$, the 'best fit', before the large gap in dimensions, can be summarised as follows: All the relevant operators appear, together with a set of irrelevant states whose $\Delta^{P}$ 's match acting on all these operators but two: the first $\left(\square \Phi_{1,1} \equiv 0\right)$ and the last $\left(\square \Phi_{p-1, p-2}\right.$ is missing). Note that the $O\left(\partial^{2}\right)$ spectrum has an unbroken oscillating pattern of $Z_{2}$ parities $(+-+-\cdots+-)$, which would have been broken for $\Delta>2$ had it also (or only) uncovered the irrelevant primaries here.

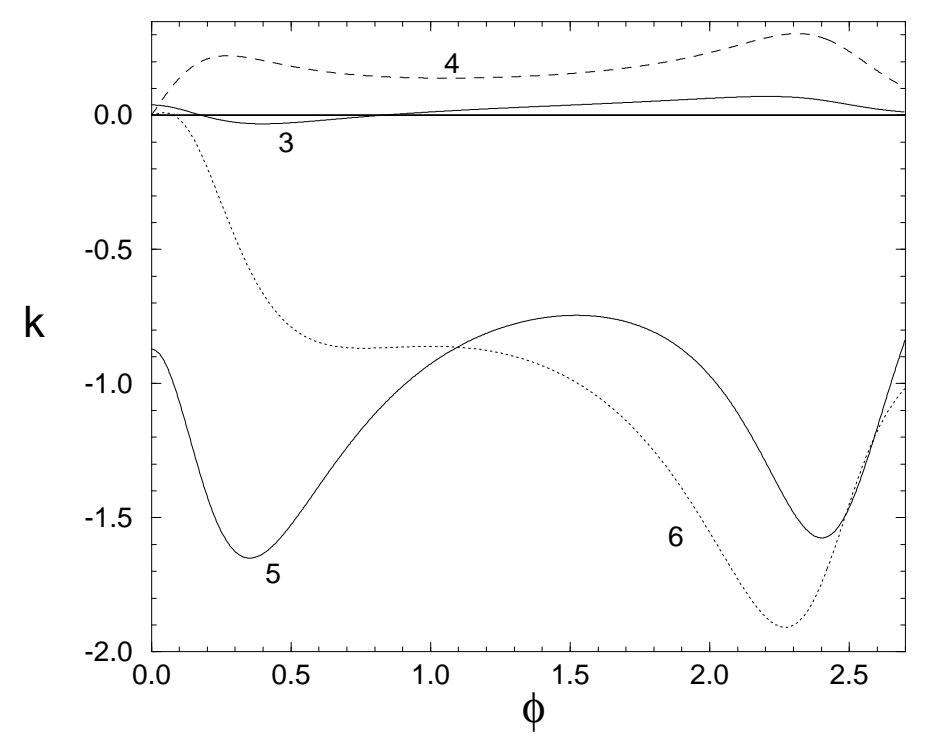

Fig.5. The $k(\varphi)$ component of the $3^{\text {rd }}-6^{\text {th }}$ eigenoperators at the tricritical Ising model fixed point $(p=4)$. Normalisations are those of fig.4. Recall that the $1^{\text {st }}$ and $2^{\text {nd }}$ operator have $k(\varphi) \equiv 0$.

We briefly mention directions for further research. Evidently, taking the derivative expansion to higher order would be informative. We should, for example, begin to see approximate degeneracies in the irrelevant part of the spectrum. Expanding the flow equations to second order in the perturbations would allow computation of the operator product (fusion) coefficients, while non-scalar couplings would allow access to operators with non-zero spin and e.g. an investigation of the approximately satisfied Virasoro symmetry. The full partial differential equations (8), analyzed e.g. again by relaxation, can describe for example flow (crossover) from one FP 
to another, using the present analysis to provide the BCs; in particular one can investigate this way the form of the bare Landau Ginsburg Lagrangian. Finally it would be interesting to consider, by relaxation, 'flow' of the FPs with (general real) dimension $D$. All of the above suggestions would help in resolving the reason for our "puzzling operators".

\section{Note added in proof.}

A natural interpretation of our puzzling operators would be that they are examples of "shadow operators" [13]. (I thank T. Hollowood for bringing this work to my attention). These are operators that exist in the continuum theory but completely decouple in the conformal invariant limit. If so, we should expect to find that, in our approximation, their fusion coefficients with CFT operators are very small.

\section{Acknowledgements}

It is a pleasure to thank John Cardy, Tim Hollowood and especially Michel Bauer for their interest, and advice on CFT and the $\varepsilon$ expansion, and to thank Martin Hasenbusch for a discussion about Monte Carlo methods.

\section{Appendix A. Numerics.}

We sketch some of the less obvious points involved in the numerical solution of (8) at, and linearized about, the FPs. Note first the reasons for relaxation: it is not necessary to cast the eqns (8) in the form $V^{\prime \prime}(\varphi)=\cdots$ and $K^{\prime \prime}(\varphi)=\cdots$; two point BCs and eigenvalue problems are easily incorporated; and it is efficient in the sort of searches illustrated in fig.1, since the previous solution is a good guess for the next[9]. It can also cope with stiffness[9], which is there because small perturbations from a solution result in singularities at some finite $\varphi$. Conditions (10) can be violated in the iterands. If this happened it worked best to back track and shorten the jumps between iterations. It is necessary to develop the asymptotic BCs (5) at least to next-to-leading order, introducing explicitly proportionality constants, say $A_{V}$ and $A_{K}$. This is because we set these BCs at some finite point $\varphi=\varphi_{A S Y}$, and imposing just (5) would imply, by (8), singular values for the second derivatives. In fact we developed the asymptotics yet one further order so as to check that the 
truncation error was well under control. $\varphi_{A S Y}$ must lie within a window bounded below by points where the asymptotic series is not a sufficiently good approximation and above by points where roundoff error (due to increasing stiffness) prevents the relaxation program from converging. This window rapidly shrinks with increasing $p$ because both effects (we believe) depend on the size of $\sim \varphi^{8 / \eta}$. We found empirically that the upper bound is just above the last turning point in $K$. This turning point always exists, so we ensure we are automatically inside this window by setting $\varphi_{A S Y}=1$ and normalising with $K_{a s y}^{\prime \prime}(1)=0$, where $K_{a s y}(\varphi)$ is the asymptotic expansion of $K$. We checked that our results are completely insensitive to moderate changes in $\varphi_{A S Y} \lesssim 1$. We factor out the rapid increase in $V$ in the asymptotic region (5), by writing $V(\varphi)=\left[V(0)+A_{V} \varphi^{2 r}\right] \tilde{V}(\varphi)$, where the integer $r \approx 2 / \eta$, the multiplying factor being differentiated analytically. Numerical errors are then under control even for $p=12$ where asymptotically $V \sim \varphi^{200}$. For the eigenvalues $\lambda$, it was enough to substitute the FP solutions into the linearized equations, using the same mesh, imposing the leading asymptotic behaviour on $v, k$ at $\varphi=\varphi_{a s y}$. In the positive[negative] parity search, we recorded those cases where $v(1)$ changes sign but $v^{\prime}(0)[v(0)]$ does not, since sometimes these are due to passing over eigenvalues, however cases where both $v^{\prime}(0)[v(0)]$ and $v(1)$ changed sign were due to $k(0)\left[k^{\prime}(0)\right]$ passing through zero. The most rapid asymptotic behaviour was factored out of both $v$ and $k$. For the search we chose $\varphi_{a s y}=1$, but for 'polishing' it was often necessary to choose $\varphi_{\text {asy }}<1$ for convergence. We required as much convergence as possible before choosing $\varphi_{\text {asy }}$ just inside the remaining wild oscillations, checking insensitivity to moderate reductions in this value. Finally next to leading order $v$ and $k$ asymptotics were used as a check particularly that no solutions were spurious. 


\section{References}

[1] A.B. Zamolodchikov, Yad. Fiz. 44 (1986) 821.

[2] J.L. Cardy, in "Phase Transitions and Critical Phenomena", vol. 11, C. Domb and J.L. Lebowitz ed. (1987) Academic Press.

[3] P.S. Howe and P.C. West, Phys. Lett. B223 (1989) 371;

M. Bauer, E. Brézin and C. Itzykson (1987), unpublished.

[4] T.R. Morris, Int. J. Mod. Phys. A9 (1994) 2411.

[5] T.R. Morris, Phys. Lett. B329 (1994) 241.

[6] T.R. Morris, Phys. Lett. B334 (1994) 355.

[7] K. Wilson and J. Kogut, Phys. Rep. 12C (1974) 75.

[8] F.J. Wegner, J. Phys. C7 (1974) 2098.

[9] W.H. Press et al, "Numerical Recipes .. The Art of Scientific Computing", 2nd edition (1992) C.U.P.

[10] A.B. Zamolodchikov, JETP Lett. 43 (1986) 730.

[11] See for example the reviews A.B. Zamolodchikov and Al.B. Zamolodchikov, Sov. Sci. Rev. A. Phys. 10 (1989) 269;

J.L. Cardy, "Conformal Invariance and Statistical Mechanics", Les Houches Summer School (1988) 169.

[12] For graphs of $V$ and $K$ at $p=12$ see T.R. Morris, in Proceedings of XII International Symposium on Lattice Field Theory, LATTICE '94, Bielefeld, Germany, Eds. F. Karsch et al, Southampton preprint SHEP 94/95-10, hep-lat/9411053.

[13] B. Schroer, Nucl. Phys. B295[FS21] (1988) 586. 